\title{
Acetilación de Aminas Alifáticas y Aromáticas: Estudio Teórico
}

\author{
Silvana C. Caglieri y Héctor R. Macaño \\ CIQA-Centro de Investigación y Transferencia en Ingeniería Química Ambiental, \\ Facultad Regional Córdoba, Universidad Tecnológica Nacional, Córdoba - Argentina \\ (e-mail: scaglieri@quimica.frc.utn.edu.ar; hmacano@quimica.frc.utn.edu.ar)
}

Recibido Sep. 1, 2015; Aceptado Nov. 4, 2015; Versión final Nov. 19, 2015, Publicado Abr. 2016

\section{Resumen}

Se realizó un estudio teórico comparativo de reactividad de metilamina, anilina, difenilamina, p-nitroanilina y p-metilanilina, frente a la reacción de acetilación, a través del análisis de los intermediarios de reacción correspondientes. La acetilación de aminas es una de las transformaciones más frecuentemente usadas en síntesis orgánica, ya que proporciona un medio eficiente y económico para proteger el grupo amino en un proceso sintético. Las energías de activación y las energías de los intermediarios de reacción se calcularon con dos niveles de teoría: teoría funcional de densidad (DFT) con el estándar B3LYP y la teoría de perturbación de Møller-Plesset (MP2) combinados con el conjunto de base 6-31G*. Los valores obtenidos se compararon con datos de literatura. La metilamina presentó la mayor reactividad frente a la acetilación y el método DFT reportó los valores más bajos de energía.

\section{Aliphatic and Aromatic Amines Acetylation: Theoretical Study}

\begin{abstract}
A comparative theoretical study of reactivity of methylamine, aniline, diphenylamine, $p$-nitroaniline and $p$ methylaniline acetylation, through the analysis of the corresponding reaction intermediates was carried out. The acetylation of amines is one of the most frequently used transformations in organic synthesis since it provides an efficient and inexpensive means for protecting amino group in a synthetic process. The activation energies and the reaction intermediates energies were calculated with two levels of theory: density functional theory (DFT) with the standard B3LYP and the Møller-Plesset perturbation theory (MP2) combined with the basis set $6-31 \mathrm{G}^{*}$. The calculated values were compared with literature data. The methylamine presented the higher reactivity in the acetylation reaction and the DFT method gave the lower energy values.
\end{abstract}




\section{INTRODUCCIÓN}

El estudio de la acetilación de aminas es de gran interés por la utilidad, dentro de la industria química, de las amidas, productos de reacción, sobre todo las aril amidas empleadas por su actividad biológica como analgésicos y antiinflamatorios (Kushwaha et al., 2011). Además constituye una de las transformaciones más frecuentemente usadas en síntesis orgánica, ya que proporciona un medio eficiente y económico para la protección del grupo amino en un proceso sintético. Han sido reportados estudios sobre empleo de amidas en la síntesis de productos químicos como por ejemplo insecticidas ambientalmente benignos (Zhao et al., 2013) e investigaciones sobre la protección del grupo amino en la síntesis de proteínas (Nilsson et al., 2005) y síntesis de péptidos (Takahashi et al., 2012).

La acetilación de una amina es una reacción de sustitución nucleofílica sobre carbono insaturado, siendo el nucleófilo la propia amina. Dicha reacción se puede llevar a cabo con cloruro de acetilo, con el agregado de una base (Yadav et al., 2001), con acetato de amonio en ácido acético, ácido de Brönsted (Prasad et al., 2005) o con el agregado de un ácido de Lewis (Farhadi y Panahandehjoo, 2010). El mecanismo de la acetilación consiste en un ataque de la amina al carbono del carbonilo del anhídrido acético, formando un intermediario tetraédrico, etapa determinante de la velocidad de reacción. Se han realizado estudios teóricos sobre la reacción de acetilación de aril aminas empleando el método DFT (Tong et al., 2012) y trabajos experimentales sobre la reacción de acetilación de diferentes aminas con anhídrido acético (Naik et al., 2004) y ambos coinciden en que la misma transcurre a través de la formación de un intermediario tetraédrico.

Para llevar a cabo el estudio teórico de acetilación de aminas y poder comparar la reactividad de las mismas frente a dicha reacción, se seleccionaron diferentes tipos de aminas: alifática, aromáticas, anilinas sustituidas, aminas primarias y amina secundaria. Al reaccionar anhídrido acético con las aminas seleccionadas, a saber: metilamina, anilina, difenilamina, $\mathrm{p}$-nitroanilina y $\mathrm{p}$-metilanilina, se obtienen como productos de reacción ácido acético y las siguientes amidas: $\mathrm{N}$-metilacetamida, $\mathrm{N}$-fenilacetamida (acetanilida), N,N-difenilacetamida (N-fenilacetanilida), N-4-nitrofenilacetamida y N-4-metilfenilacetamida, respectivamente. Este estudio aporta nuevos datos a la reacción de acetilación de aminas en particular y a la química orgánica en general, empleando la química teórica como herramienta para analizar las estructuras y propiedades de diferentes compuestos orgánicos y predecir comportamientos de reacciones químicas en menor tiempo y mayor seguridad.

\section{METODOLOGÍA}

Para poder comparar la reactividad de las aminas frente a la acetilación, se diseñaron y optimizaron las estructuras de todas las especies que intervienen en las reacciones, determinándose los parámetros geométricos óptimos correspondientes, calculando las energías mínimas de todos los compuestos que participan en las reacciones, como así también las energías de los respectivos intermediarios tetraédricos.

Para realizar estos cálculos se emplearon métodos de estructura electrónica. Estos métodos dentro de la química computacional, utilizan las leyes de la mecánica cuántica como base para los cálculos, mediante la resolución de la ecuación de Schrödinger, que independiente del tiempo toma la forma de la siguiente ecuación:

$H \Psi_{(r)}=E \Psi_{(r)}$

Siendo $\mathrm{H}$ una función diferencial que incluye la energía cinética y potencial de núcleos y electrones, denominada operador hamiltoniano, $\Psi$ la función de onda molecular y E energía. La solución que obtiene el menor valor de energía es la que corresponde al estado basal.

Dentro de los métodos de estructura electrónica, se empleó el método DFT basado en la teoría de funcionales de densidad (Parr y Yang, 1989), el cual incluye efectos de correlación electrónica y dentro de este se utilizó el B3LYP (Becke, 1997). Este método se ha empleado para calcular las energías de activación de la hidrólisis de diferentes amidas, siendo esta reacción también una sustitución nucleofílica sobre carbonilo (Caglieri y Pagnan, 2013).

Además se empleó el método MP2, basado en la teoría perturbativa de Møller-Plesset de segundo orden. Este método denominado ab-initio, considera toda la estructura atómica sin introducir parámetros empíricos en la resolución de la ecuación de Schrödinger.Se optó por el conjunto de base 6-31G* que indica que se utilizan seis funciones gaussianas para representar los orbitales atómicos internos, mientras que los externos, capa de valencia, se representan mediante tres funciones para la parte contraída y una para la 
parte difusa, más una función de polarización para átomos pesados, indicada mediante el *, que representa el efecto de los orbitales tipo d. Todos los cálculos se realizaron empleando el programa Gaussian'09 (Frisch et al., 2009).

\section{RESULTADOS Y DISCUSIÓN}

El esquema general de la acetilación de metilamina $\left(R=\mathrm{CH}_{3}, \mathrm{R}_{1}=\mathrm{H}\right)$, anilina $\left(\mathrm{R}=\mathrm{C}_{6} \mathrm{H}_{5}, \mathrm{R}_{1}=\mathrm{H}\right)$, difenilamina $\left(R=C_{6} H_{5}, R_{1}=C_{6} H_{5}\right)$, p-nitroanilina $\left(R=C_{6} H_{4} N_{2}, R_{1}=H\right)$ y $p$-metilanilina $\left(R=C_{7} H_{7}, R_{1}=H\right)$ se muestra en la Fig.1, siendo los productos de reacción ácido acético, $N$-metilacetamida $\left(R=C_{3}, R_{1}=H\right)$, acetanilida $\left(\mathrm{R}=\mathrm{C}_{6} \mathrm{H}_{5}, \mathrm{R}_{1}=\mathrm{H}\right)$, N-fenilacetanilida $\left(\mathrm{R}=\mathrm{C}_{6} \mathrm{H}_{5}, \mathrm{R}_{1}=\mathrm{C}_{6} \mathrm{H}_{5}\right), \mathrm{N}$-4-nitrofenilacetamida $\left(\mathrm{R}=\mathrm{C}_{6} \mathrm{H}_{4} \mathrm{NO}_{2}, \mathrm{R}_{1}=\mathrm{H}\right)$ y N-4metilfenilacetamida $\left(R=\mathrm{C}_{7} \mathrm{H}_{7}, \mathrm{R}_{1}=\mathrm{H}\right)$, respectivamente.<smiles>[R]N[CH-][I-]N([R7])[3H]</smiles>

Fig.1: Esquema general de la reacción de acetilación

La Tabla 1 muestra los resultados obtenidos de la aplicación de los métodos de cálculo mencionados, es decir en la misma se reportan las energías de los reactivos que intervienen en cada una de las reacciones motivo de estudio, a saber: metilamina, anilina, difenilamina, p-nitroanilina, p-metilanilina y anhídrido acético.

Tabla 1: Estructuras y energías de reactivos

\begin{tabular}{|c|c|c|c|}
\hline Reactivos & Estructuras & Energía (Hartree) - DFT & Energía (Hartree)-MP2 \\
\hline Metilamina & & -95.8350 & -95.3844 \\
\hline Anilina & & -287.5518 & -286.2771 \\
\hline Difenilamina & & -518.5165 & -516.2142 \\
\hline p-nitroanilina & & -491.9437 & -489.9896 \\
\hline p-metilanilina & & -326.8386 & -325.3657 \\
\hline Anhídrido Acético & & -381.6166 & -380.1577 \\
\hline
\end{tabular}

En la Fig.2 se describen las etapas a través de las cuales se lleva a cabo la reacción de acetilación de metilamina $\left(R=\mathrm{CH}_{3}, \mathrm{R}_{1}=\mathrm{H}\right)$, anilina $\left(R=\mathrm{C}_{6} \mathrm{H}_{5}, \mathrm{R}_{1}=\mathrm{H}\right)$, difenilamina $\left(R=\mathrm{C}_{6} \mathrm{H}_{5}, \quad \mathrm{R}_{1}=\mathrm{C}_{6} \mathrm{H}_{5}\right)$, p-nitroanilina $\left(\mathrm{R}=\mathrm{C}_{6} \mathrm{H}_{4} \mathrm{NO}_{2}, \mathrm{R}_{1}=\mathrm{H}\right)$ y $\mathrm{p}$-metilanilina $\left(\mathrm{R}=\mathrm{C}_{7} \mathrm{H}_{7}, \mathrm{R}_{1}=\mathrm{H}\right)$. 
<smiles>[R]N[R]CCCCCC(C)=O</smiles><smiles>[R][NH+]([R])C(C)=O</smiles><smiles>[R7][N+]([R7])(CC)C(C)(C)OC(C)=O</smiles><smiles>[R]N([18F])C(C)=O</smiles>

Fig. 2: Mecanismo de la reacción de acetilación

El mecanismo de la reacción de acetilación consiste en una primera etapa del ataque nucleofílico de la amina al carbono del carbonilo del anhídrido acético, gracias a la polarización que sufre dicho grupo, formando el intermediario tetraédrico, indicado entre corchetes en la Fig.2. Posteriormente se re-establece el doble enlace carbono -oxígeno y se elimina el anión acetato como grupo saliente que actúa como base sustrayendo un protón de la amida protonada, generando de esta manera la amida producto de reacción. Estudios sobre mecanismos de reacción coinciden en considerar al intermediario tetraédrico, como el estado de transición y la etapa determinante de la velocidad de reacción de la sustitución nucleofílica sobre carbonilo (Bruckner, 2001). Para simplificar el modelo los efectos del solvente no fueron considerados.

La Tabla 2 muestra los resultados obtenidos de la aplicación de los métodos de cálculo mencionados, es decir en la misma se reportan las energías de los intermediarios tetraédricos de cada una de las acetilaciones motivo de estudio.

Tabla 2: Estructuras y energías de los intermediarios de reacción

\begin{tabular}{|c|c|c|c|}
\hline Intermediarios & Estructuras & $\begin{array}{c}\text { Energía (Hartree) } \\
\text { DFT }\end{array}$ & $\begin{array}{c}\text { Energía (Hartree) } \\
\text { MP2 }\end{array}$ \\
\hline $\begin{array}{l}\text { Acetilación de } \\
\text { metilamina }\end{array}$ & & -477.4395 & -475.5262 \\
\hline $\begin{array}{l}\text { Acetilación de } \\
\text { anilina }\end{array}$ & & -669.1404 & -666.4038 \\
\hline $\begin{array}{l}\text { Acetilación } \\
\text { difenilamina }\end{array}$ & & -900.1044 & - 896.3399 \\
\hline $\begin{array}{c}\text { Acetilación } \\
\text { p-nitroanilina }\end{array}$ & & -873.5262 & -870.1106 \\
\hline $\begin{array}{c}\text { Acetilación } \\
\text { p-metilanilina }\end{array}$ & & -708.4348 & -705.5011 \\
\hline
\end{tabular}

Los valores de energías de activación $(E A=E I$ - Ereactivos) calculadas empleando ambos métodos se reportan en la Tabla 3, siendo El la energía del intermediario tetraédrico y Ereactivos la suma de las energías del anhídrido acético y la amina correspondiente. Conversión 1 Hartree equivale a $627.51 \mathrm{kcal} / \mathrm{mol}$. 
El método DFT reportó valores de energías de activación más bajos, para todas las acetilaciones, en comparación con el método MP2. Para la acetilación de metilamina se obtuvo una energía de activación (EA) de $9.98 \mathrm{kcal} / \mathrm{mol}$ empleando el método MP2 y estudios teóricos utilizando dicho método reportan valores de energía de activación de $9 \mathrm{kcal} / \mathrm{mol}$ (Kruger, 2002) para dicha reacción.

Para la acetilación de anilina se obtuvo un valor de energía de activación (EA) de $17.57 \mathrm{kcal} / \mathrm{mol}$ con DFT. Estudios teóricos, aplicando DFT y estudios experimentales han reportado que la energía de activación de la acetilación de anilina supera en aproximadamente $10 \mathrm{kcal} / \mathrm{mol}$ a la de la metilamina (Tong et al., 2012).

Tabla 3: Energías de activación de las acetilaciones

\begin{tabular}{|c|c|c|}
\hline Acetilación & $\begin{array}{c}\text { Energía de activación (Kcal/mol) } \\
\text { DFT }\end{array}$ & $\begin{array}{c}\text { Energía de activación (Kcal/mol) } \\
\text { MP2 }\end{array}$ \\
\hline metilamina & 7.59 & 9.98 \\
\hline anilina & 17.57 & 19.45 \\
\hline difenilamina & 18.01 & 20.08 \\
\hline p-nitroanilina & 21.39 & 23.03 \\
\hline p-metilanilina & 12.80 & 13.99 \\
\hline
\end{tabular}

La menor reactividad de la anilina frente a la acetilación en comparación con la metilamina, se puede asociar al reemplazo de un grupo metilo por un grupo fenilo, más voluminoso, provocando este último un mayor impedimento estérico que se traduce en un aumento en la energía de activación.

Se observa que la difenilamina, frente a la acetilación con DFT, es $0.44 \mathrm{kcal} / \mathrm{mol}$ y con MP2, $0.63 \mathrm{kcal} / \mathrm{mol}$, menos reactiva que la anilina, esto se asocia al agregado de otro grupo fenilo, que gracias a su planaridad, ambos conforman una estructura con un impedimento estérico menor que el esperado, que se traduce en una pequeña diferencia entre las energías de activación y de reactividad.

La menor reactividad de la anilina frente a la acetilación en comparación con la metilamina se asocia al reemplazo de un grupo metilo por un grupo fenilo, este último permite o favorece la deslocalización en el anillo aromático de los electrones no enlazados del nitrógeno de la amina, disminuyendo su nucleofilidad y dificultando el ataque de la misma al carbono del carbonilo del anhídrido acético. Por el mismo motivo, al reemplazar otro hidrógeno por otro grupo fenilo disminuye la nucleofilidad de la difenilamina que resulta ser menos reactiva en comparación con la anilina, reportando los cálculos a nivel teórico un valor de energía de activación (EA) de $18.01 \mathrm{kcal} / \mathrm{mol}$ para dicha reacción con DFT.

La p-nitroanilina presenta una menor reactividad frente a la acetilación comparada con la anilina, esto puede asociarse al efecto de atracción de electrones ejercido por el grupo nitro sobre el anillo aromático, por diferencia de electronegatividad, que favorece la deslocalización electrónica, en el anillo aromático, de los electrones no enlazados del nitrógeno de la amina, disminuyendo la nucleofilidad de la misma (Prasad et al., 2005; Aerry et al., 2013).

La p-metilanilina muestra una mayor reactividad frente a la acetilación comparada con la anilina, esto puede asociarse al efecto dador de electrones ejercido por el grupo metilo sobre el anillo aromático, debido al efecto inductivo por diferencia de hibridación $\left(\mathrm{sp}^{3} \rightarrow \mathrm{sp}^{2}\right)$, que favorece la disposición electrónica por parte del nitrógeno de la amina, aumentando su nucleofilidad (Naik et al., 2004; Aerry et al., 2013).

\section{CONCLUSIONES}

De acuerdo a los resultados y discusión presentados en este trabajo, se pueden obtener las siguientes conclusiones:

i) Se reportó un orden de reactividad para las aminas: metilamina > p-metilanilina $>$ anilina $>$ difenilamina $>$ p-nitroanilina, frente a la acetilación.

ii) El método DFT con la combinación B3LYP/6-31G* describe correctamente la sustitución nucleofílica sobre carbono insaturado o carbonilo.

iii) La existencia de una marcada concordancia entre los resultados teóricos obtenidos y los datos de literatura, valida el empleo de métodos teóricos como herramientas para el estudio del sistema químico planteado. 


\section{REFERENCIAS}

Aerry, S., A. Kumar, A. Saxena, A. De y S. Mozumdar, Chemoselective acetylation of amines and thiols using monodispersed Ni-nanoparticles, doi:10.1080/17518253.2012.737029, Green Chem. Lett. Rev.: 6(2), 183-188 (2013)

Becke, A.D., Density-functional thermochemistry V. Systematic optimization of exchange-correlation functionals, J. Chem. Phys.: 107, 8554-8560 (1997)

Bruckner, R., Advanced Organic Chemistry Reaction Mechanisms, 224-244. Elsevier Science Publishing Co Inc., Freiburg, Alemania (2001)

Caglieri, S.C. y M. Pagnan, Estudio Teórico sobre la Hidrólisis Ácida de Amidas Alifáticas y Aromáticas, doi: 10.4067/S0718-07642013000300005, Inf.Tecnol. (en línea), 24(3), 35-40 (2013)

Farhadi, S. y S. Panahandehjoo, Spinel-Type Zinc Aluminate $\left(\mathrm{ZnAl}_{2} \mathrm{O}_{4}\right)$ Nanoparticles Prepared by the CoPrecipitation Method: A Novel, Green and Recyclable Heterogeneous Catalyst for the Acetylation of Amines, Alcohols and Phenols under Solvent-Free Conditions, Appl. Catal., A: 382, 293-302 (2010)

Frisch, M.J., G. W. Trucks y otros 71 autores, Gaussian 09, Revision D.01, 2ª edición. Gaussian, Inc., Wallingford CT, USA (2009)

Kruger, H.G., Ab initio mechanistic study of the protection of alcohols and amines with anhydrides, J. Mol. Struct.: 577, 281- 285 (2002)

Kushwaha, N., R. K. Saini y S.K.S. Kushwaha, Synthesis of some Amide derivatives and their Biological activity, Int. J. ChemTech Res.: 3 (1), 203-209 (2011)

Naik, S., G. Bhattacharjya, B. Talukdar y B.K. Patel, Chemoselective Acylation of Amines in Aqueous Media, Eur. J. Org. Chem.: 6, 1254-1260 (2004)

Nilsson, B.L., M. B. Soellner y R.T. Raines, Chemical Synthesis of Proteins, Annu. Rev. Biophys. Biomol. Struct.: 34, 91-118 (2005)

Parr, R.G. y W. Yang, Density-functional theory of atoms and molecules, Oxford University Press.,Nueva York, USA (1989)

Prasad, H.S., G.R. Srinivasa y D. Channe Gowda, Convenient, Cost-Effective, and Mild Method for the Nacetylation of Anilines and Secondary Amines, Synth. Commun.: 35, 1189-1195 (2005)

Takahashi, D., T. Yano y T. Fukui, Novel diphenylmethyl-Derived Amide Protecting Group for Efficient Liquid-Phase Peptide Synthesis: AJIPHASE, Org. Lett.: 14(17), 4514-4517(2012)

Tong, X., Z. Ren, X. Qü, Q. Yang y W. Zhang, Efficient amide formation from arylamines and esters promoted by $\mathrm{AlCl}_{3} / \mathrm{Et}_{3} \mathrm{~N}$ : an experimental and computational investigation, Res. Chem. Intermed.: 38, 19611968 (2012)

Yadav, V.K., K. G. Babu y M. Mittal, $\mathrm{KF}^{-} \mathrm{Al}_{2} \mathrm{O}_{3}$ is an Efficient Solid Support Reagent for the Acetylation of Alcohols and Amines. Impeding Effect of Solvent on the Reaction Rate, Tetrahedron: 57, 7047-7051(2001)

Zhao, Y., Y. Li, L. Xiong, L.Xu, L.Peng, F. Li y Z. Li, Design, syntheses and biological activities of novel anthranilic diamide insecticides containing N-pyridylpyrazole, Chem. Res. Chin. Univ.: 29, 51-56 (2013) 